Short Report

\title{
Direct Evidence that Left Ventricular Myocardium Is Incompressible Throughout Systole and Diastole
}

\author{
Kai Tsuiki and ERIK L. Ritman* \\ The First Department of Internal Medicine, Yamagata \\ University School of Medicine, Yamagata 990-23 and \\ *Biodynamics Research Unit. Mayo Clinic, Rochester. \\ Minnesota 55901, U.S.A.
}

Tsuiki, K. and Ritman, E.L. Direct Evidence that Left Ventricular Myocardium Is Incompressible Throughout Systole and Diastole. Tohoku J. exp. Med., 1980, 132 (1), 119-120 - Incompressibility of left ventricular myocardium was tested utilizing isolated canine working left ventricular preparations. This ventricle functioned at levels comparable to the intact heart. The ventricular muscle volume was calculated with the videometric border recognition technique, which is probably the most satisfactory among the presently available techniques to measure left ventricular volume. The calculated wall mass throughout the cardiac cycle varied ranging between about $\pm 5 \%$. The difference between the calculated and weighed myocardial mass was within $\pm 7 \%$ of the true weight. These findings warrant the idea that myocardial muscle mass is incompressible throughout systole and diastole; the assumption used to calculate some variables of left ventricular function._ videometry; isolated working left ventricle

Mirsky (1974) stated that most biological tissues are incompressible and the myocardium in particular may be classified as an incompressible, anisotropic, nonhomogeneous, elastic material. Assumption of incompressibility is fundamental in order to calculate several indices of ventricular performance. For example, left ventricular mass may be calculated from end-diastolic wall thickness and radius, assuming that myocardial mass remains constant during systole and diastole. Hugenholz, Kaplan and Hull (1969) developed a method permitting the prediction of wall thickness on any film taken during cardiac cycle, since angiographic recognition of the left ventricular endocardial border had been usually difficult except those of end-diastole. In this approach, end-diastolic muscle mass was considered the most accurate and its volume was assumed not to change during systole. However, whether myocardium is incompressible or not has not been well demonstrated. That skeletal muscle is practically incompressible during contraction has been accurately shown (Abott and Baskin 1962). Utilizing a sophisticated technique which permitted the measurement of small, rapid volume changes occurring in a muscle during a twitch, they reported in the sartorius muscle that the transient increase which commences at between 2 and $3 \mathrm{msec}$ after the stimulus is about $2.5 \times 10^{-6}$ and that the decrease during a twitch is about $5 \times 10^{-6} \mathrm{ml} / \mathrm{g}$.

We measured the wall volume of the canine isolated working left ventricle using videoventriculography (Tsuiki et al. 1973). This ventricle was metabolically supported and functioned at levels comparable to the intact heart (Ritman 1975). In these preparations the biplane images of the ventricle were recorded on one videotape at 60 times/sec. Wall mass was calculated as the difference between calculated volume encompassed by the epicardium and the volume encompassed by the endocardium. Each volume was calculated according to Simpson's rule. The regression equation relating stroke volume determined by angiography $(Y)$ and that determined volumetrically with a graduated cylinder $(X)$ for 6 preparations was $Y=1.04 X-0.3, r=0.99$ (Ritman 1975), indicating that the videometric border recognition was quite accurate throughout diastole to systole as far as the endo-

Received for publication, December 4, 1979. 


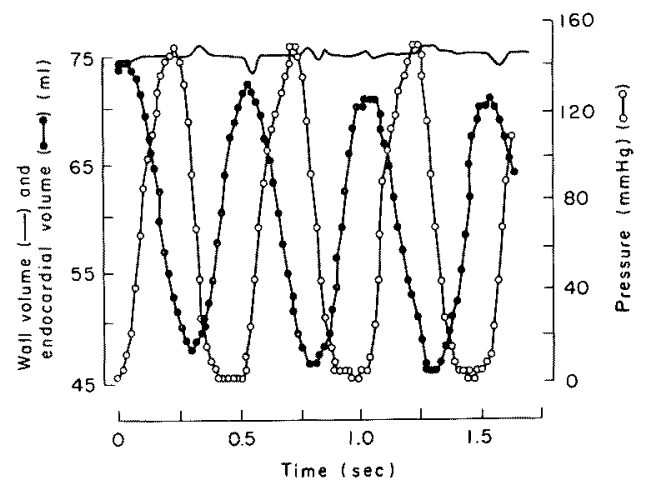

Fig. 1. Volume of the left ventricular wall computed from biplane video angiograms of the metabolically supported isolated canine left ventricle (muscle weight: $81.5 \mathrm{~g}$ ). The endocardial volume (indicated by solid circles) and the simultaneously recorded left ventricular pressure (open circles) are also shown.

cardial border was concerned. Fig. 1 shows computer-generated plot of volume of the left ventricular myocardium (wall mass). Also shown are the volume contained by the endocardial outline (solid circles) and the plot of the simultaneously recorded left ventricular pressure (open circles). The calculated wall mass throughout the cardiac cycle varied about $\pm 5 \%$ in this particular case as was found in the other ventricles studied - mostly a transient decrease at the onset of systole and an increase at the end of diastole. This order of the variation is comparable to the error when the cardiac volume is calculated from the various angles of view during the same phase of the cardiac cycle over $180^{\circ}$ rotation of ventricular cast (Ritman, Sturm and Wood 1971). Ventricular muscle mass weight was $90 \pm 10 \mathrm{~g}$ ( \pm S.D.) on an average and the difference between the calculated and weighed myocardial mass was within $\pm 7 \%$ of the true weight. It should be also recognized that a part of blood which is contained in the diastolic ventricular wall may be squeezed out during systole. The amount at a maximum should be $6-8 \%$ of tissue mass (Gibson et al. 1946). The variation in the calculated wall volume as shown coincides with the possible change in coronary blood volume during each phase of the cardiac cycle. Nonetheless, since data from silastic casts of ventricular chambers indicate a probable uncertainty of $\pm 8 \%$ in absolute value (Ritman, Sturm and Wood 1970), the method may not be accurate beyond this order of error, but the method is probably the most satisfactory among the presently available techniques to measure left ventricular volume.

\section{References}

1) Abott, B.C. \& Baskin, R.J. (1962) Volume changes in frog muscle during contraction. J. Physiol., 161, 379-391.

2) Gibson, J.G., Seligman, A.M., Peacock, W.C., Aub, J.C., Fine, J. \& Evans, R.D. (1946) The distribution of red cells and plasma in large and minute vessels of the normal dog, determined by radioactive isotopes of iron and iodine. J. clin. Invest., 25, 848-857.

3) Hugenholz, P.G., Kaplan, E. \& Hull, E. (1969) Determination of left ventricular wall thickness by angiocardiography. Amer. Heart $J ., 78,513-522$.

4) Mirsky, I. (1974) Basic terminology and formulae for left ventricular wall stress. In: Cardiac Mechanics, edited by I. Mirsky, D.N. Ghista \& H. Sandler, John Wiley \& Sons Inc., New York-London-Sydney-Toronto, pp. 3-10.

5) Ritman, E.L., Sturm, R.E. \& Wood, E.H. (1970) Comparison of volume of canine left ventricular casts and angiograms using biplane and monoplane roentgen videometry. Physiologist, 13, 294.

6) Ritman, E.L., Sturm, R.E. \& Wood, E.H. (1971) A biplane roentgen videometry system for dynamic $(60 / \mathrm{sec})$ studies of the shape and size of circulatory structures, particularly the left ventricle. In: Roentgen-, Cine-, and Video-Densitometry: Fundamentals and Applications for Blood Flow and Heart Volume Determination, G. Thieme Verlag (Heintzen ed.), Stuttgart, pp. 179-211.

7) Ritman, E.L. (1975) Left ventricular function and myocardial contractility: Analysis by roentgen videoangiographic techniques. Mayo Clinic Proc., 50, 147-156.

8) Tsuiki, K., Ritman, E.L., Donald, D.E., Sturm, R.E. \& Wood, E.H. (1973) Videometric determination of wall dynamics in a working isolated canine left ventricle. Physiologist, 16, 473 . 